GA-A21903

\title{
EFFECT OF \\ TOROIDAL PLASMA FLOW \\ AND FLOW SHEAR \\ ON GLOBAL MHD MODES
}

\author{
by \\ M.S. CHU, J.M. GREENE, T.H. JENSEN, \\ R.L. MILLER, A. BONDESON, R.W. JOHNSON, \\ and M.E. MAUEL
}

JANUARY 1995 


\section{DISCLAIMER}

This report was prepared as an account of work sponsored by an agency of the United States Government. Neither the United States Government nor any agency thereof, nor any of their employees, make any warranty, express or implied, or assumes any legal liability or responsibility for the accuracy, completeness, or usefulness of any information, apparatus, product, or process disclosed, or represents that its use would not infringe privately owned rights. Reference herein to any specific commercial product, process, or service by trade name, trademark, manufacturer, or otherwise does not necessarily constitute or imply its endorsement, recommendation, or favoring by the United States Government or any agency thereof. The views and opinions of authors expressed herein do not necessarily state or reflect those of the United States Government or any agency thereof. 


\section{DISCLAIMER}

Portions of this document may be illegible in electronic image products. Images are produced from the best available original document. 


\title{
EFFECT OF \\ TOROIDAL PLASMA FLOW \\ AND FLOW SHEAR \\ ON GLOBAL MHD MODES
}

\author{
by \\ M.S. CHU, J.M. GREENE, T.H. JENSEN, \\ R.L. MILLER, A. BONDESON,* R.W. JOHNSON,** \\ and M.E. MAUEL***
}

This is a preprint of an invited paper presented at the 1994 American Physical Society Division of Plasma Physics Meeting, November 7-11, 1994, Minneapolis, Minnesota, and to be printed in the Proceedings.

\author{
Work supported by \\ U.S. Department of Energy \\ Contract DE-AC03-89ER51114
}

\author{
*Uppsala University \\ **Furman University \\ ***Columbia University
}

GENERAL ATOMICS PROJECT 3940
JANUARY 1995 


\title{
Effect of Toroidal Plasma Flow and Flow Shear on Global MHD Modes*
}

\author{
M.S. Chu, J.M. Greene, T.H. Jensen, R.L. Miller, \\ A. Bondeson, ${ }^{\dagger}$ R.W. Johnson, ${ }^{\ddagger}$ and M.E. MAUEL ${ }^{\sharp}$ \\ General Atomics \\ San Diego, California 92186-9784, U.S.A.
}

\begin{abstract}
The effect of a subsonic toroidal flow on the linear magnetohydrodynamic stability of a tokamak plasma surrounded by an external resistive wall is studied. A complex non-self-adjoint eigenvalue problem for the stability of general kink and tearing modes is formulated, solved numerically, and applied to high $\beta$ tokamaks. Results indicate that toroidal plasma flow, in conjunction with dissipation in the plasma, can open a window of stability for the position of the external wall. In this window, stable plasma beta values can significantly exceed those predicted by the Troyon scaling law with no wall. Computations utilizing experimental data indicate good agreement with observations.
\end{abstract}

\footnotetext{
*This is a report of work sponsored by the U.S. Department of Energy under Contract No. DE-AC03-89ER51114.

† Uppsala University, Uppsala, Sweden.

${ }^{\ddagger}$ Furman University, Greenville, South Carolina.

${ }^{\sharp}$ Columbia University, New York City, New York.
} 


\section{Contents}

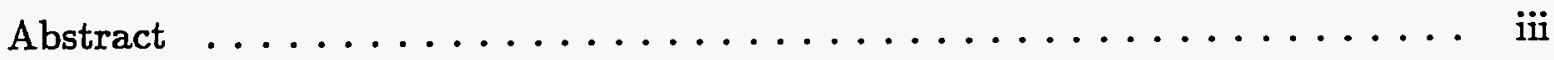

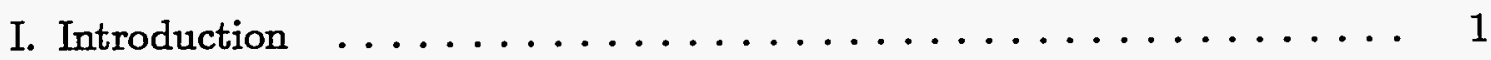

II. Formulation and Method of Solution $\ldots \ldots \ldots \ldots \ldots \ldots \ldots$

III. Physical Characteristics of the Problem $\ldots \ldots \ldots \ldots \ldots \ldots \ldots$

IV. Numerical Results . . . . . . . . . . . . . . . . 13

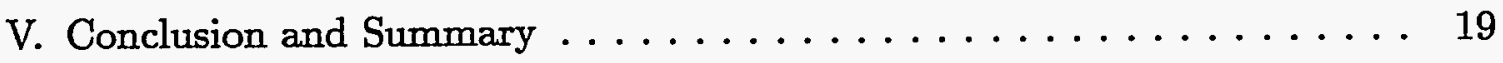

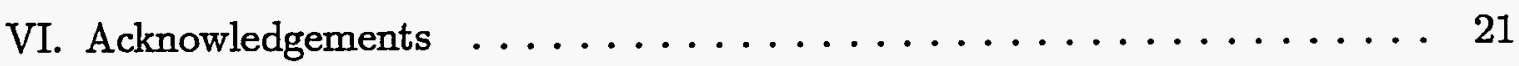

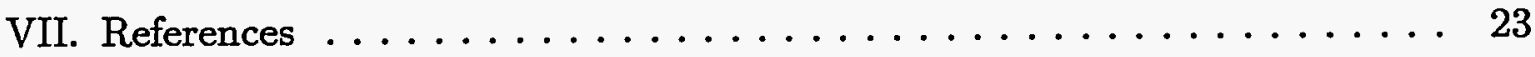

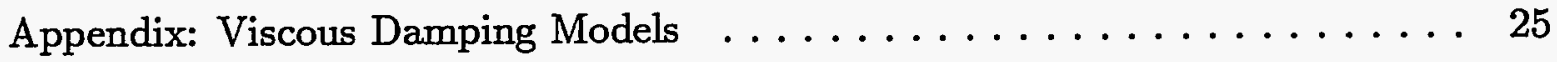

\section{Figures}

1a. Growth rate as a function of external wall location for coupled tearing mode and diffusion through the resistive wall, Eq. (17) $\ldots \ldots 99$

1b. Same as Fig. 1a for the case of $\tau_{\eta} \gg \tau_{\mathrm{w}} \ldots \ldots \ldots \ldots \ldots \ldots \ldots$

2. Growth rate as a function of the external wall location for coupled external kink and diffusion through the resistive wall, Eq. (18) $\ldots \ldots \ldots 11$

3. Growth rate diagram computed for a low edge $q$ configuration $\ldots \ldots \ldots 14$

4. Stability diagram for the same equilibrium shown in Fig. 3 with different rotation frequency and plasma dissipation $\ldots \ldots \ldots \ldots \ldots$

5. Growth rate plotted as a function of the rotation frequency of the $q=2$ surface for rotation profiles with and without rotation shear $\ldots \ldots 16$

6. Stability diagram for a high edge $q$ configuration, simulating a discharge in DIII-D . . . . . . . . . . . . . . . 17

7. Stability diagram for the same equilibrium as shown in Fig. 6 with fitted profiles to experimental rotation and density profiles $\ldots \ldots \ldots 18$ 


\section{Introduction}

In the next generation of steady-state advanced tokamaks, a high performance plasma must remain stable over time scales long compared to the flux diffusion time of the external resistive wall $\left(\tau_{\mathrm{w}}\right)$. At the same time, it is highly desirable to have $\beta_{\mathrm{N}}(\beta a B / I)$ exceed the value $\left(\beta_{\mathrm{N}}^{\mathrm{T}}\right)$ given by the Troyon scaling law with no wall. The question is: can we rely on wall stabilization for maintaining the stable operation of the plasma? It is well known ${ }^{1,2}$ that for a stationary plasma, an external resistive wall does not affect its stability, only its growth rate which is slowed down to $\sim 1 / \tau_{\mathrm{w}}$. Although the idea of resistive wall stabilization of tearing modes ${ }^{3}$ in a rotating tokamak has been around for some time, the stabilization of the external kink mode by a resistive wall for a plasma with flow was only recently proposed by Bondeson and Ward. ${ }^{4}$ In their work, they demonstrated this idea by modeling a stationary plasma surrounded by a rotating external wall.

Experimentally, it has been demonstrated in DIII-D $\mathrm{D}^{5}$ that a high beta plasma can be maintained at $\beta$ values above that given by $\beta_{\mathrm{N}}^{\mathrm{T}}$ for time scales much longer than $\tau_{\mathrm{w}}{ }^{6}$ These discharges are usually heated by co-injected neutral beams and are therefore rotating toroidally. Computations ${ }^{7}$ assuming static plasma equilibrium give critical $\beta_{\mathrm{N}}$ in agreement with experimentally observed values, when the external wall is assumed to be infinitely conducting. When the plasma slows down, instabilities are observed. The theory used in Ref. 7 ignores the toroidal rotation of the tokamak and cannot explain why the wall acts as though it were infinitely conducting. Similarly, the behavior of tearing mode unstable plasmas have been found to be at variance ${ }^{8}$ with magnetohydrodynamic (MHD) theory based on a static plasma. For instance, the plasma has been found to disrupt less frequently than suggested by the simple idea of overlapping static islands. ${ }^{9}$ These observations suggest that a comprehensive MHD study including the effect of plasma flow is needed.

The effect of flow in MHD attracted early attention from theorists. ${ }^{10}$ However, since experimentally observed flows are usually much slower than the central sound speed, it was reasoned that flow would not affect the short time scale stability of the plasma. Renewed interest has arisen recently because of the above experimental observations and the realized importance of improved stability for future tokamak development. 
This paper provides a theoretical framework for studying the stability of a tokamak plasma with a subsonic toroidal rotation and assesses its relevance to present day experiments. In Section II, we give the formulation for determining stability as a non-self-adjoint eigenvalue problem. It is solved numerically using an extension of the MARS ${ }^{11}$ code. In Section III, we present the physical problem under investigation and utilize two simple dispersion relations to elucidate the characteristics of the solutions. It is shown that, in simplified situations, a tearing mode unstable plasma surrounded by an external resistive wall satisfies a quadratic dispersion relation; while a kink unstable plasma satisfies a cubic equation. Plasma rotation coupled with dissipation can stabilize these instabilities. In Section IV, we present the numerical results for both a low $q_{\mathrm{e}}$ (edge safety factor) and a high $q_{\mathrm{e}}$, high $\beta$ equilibrium. For the high $q_{\mathrm{e}}$ equilibrium that simulates a DIII-D discharge, the computed critical rotation velocity for plasma stability agrees well with the experimental value. Section V contains a brief summary and conclusion. 


\section{Formulation and Method of Solution}

In this section we present the formulation for determination of the linear stability of a tokamak plasma with a subsonic toroidal flow and surrounded by an external resistive wall.

We start with the MHD equations for the density $\rho$, pressure $p$, magnetic field $\vec{B}$, and fluid velocity $\vec{v}$,

$$
\begin{aligned}
& \frac{d \rho}{d t}+\rho \vec{\nabla} \cdot \vec{v}=0 \\
& \frac{d p}{d t}+\Gamma p \vec{\nabla} \cdot \vec{v}=0, \\
& \frac{d \vec{B}}{d t}=(\vec{B} \cdot \vec{\nabla}) \vec{v}-(\vec{\nabla} \cdot \vec{v}) \vec{B}-\vec{\nabla} \times(\eta \vec{J}), \\
& \rho \frac{d \vec{v}}{d t}=-\vec{\nabla} p+\vec{J} \times \vec{B}-\vec{\nabla} \cdot \stackrel{\leftrightarrow}{\Pi},
\end{aligned}
$$

where

$$
\frac{d}{d t}=\frac{\partial}{\partial t}+\vec{v} \cdot \vec{\nabla}
$$

$\Gamma$ is the ratio of specific heats, $\eta$ is the plasma resistivity, and $\stackrel{\leftrightarrow}{\Pi}$ is the viscous stress tensor. At equilibrium $(\partial / \partial t=0)$, we assume that transport induced effects from $\eta$ and $\stackrel{\leftrightarrow}{\Pi}$ are negligible or implicitly balanced by sources. We also assume the plasma to have a subsonic toroidal flow

$$
\rho \vec{v}_{0} \cdot \vec{\nabla} \vec{v}_{0} \ll \vec{\nabla} p_{0}
$$

where the subscript 0 is used to denote equilibrium quantities. It is then easy to see that the the axisymmetric plasma equilibrium still satisfies the Grad-Shafranov equation 


$$
\Delta^{*} \psi_{0}=-\left(\mu_{0} R^{2} p_{0}^{\prime}+T_{0} T_{0}^{\prime}\right)
$$

and

$$
\vec{B}_{0}=T_{0} \vec{\nabla} \phi+\vec{\nabla} \phi \times \vec{\nabla} \psi_{0}
$$

In steady-state, Eq. (3) shows that the toroidal rotation frequency $\Omega_{0}\left(\psi_{0}\right)$ has to be a flux function

$$
\vec{v}_{0}=R^{2} \Omega_{0}\left(\psi_{0}\right) \vec{\nabla} \phi
$$

A straightforward linearization of Eqs. (1) through (4) shows that the density perturbation is decoupled from the rest of the system. The system of linearized equations is then

$$
\begin{aligned}
\left(\tilde{\gamma}+\underline{i n \Omega_{0}}\right) p_{1} & =-\left(\vec{v}_{1} \cdot \vec{\nabla}\right) p_{0}-\Gamma p_{0} \vec{\nabla} \cdot \vec{v}_{1} \\
\left(\tilde{\gamma}+\underline{i n \Omega_{0}}\right) \vec{b}_{1} & =\vec{\nabla} \times\left(\vec{v}_{1} \times \vec{B}_{0}-\eta \vec{\jmath}_{1}\right)+\underline{\left(\vec{b}_{1} \cdot \vec{\nabla} \Omega_{0}\right) R^{2} \vec{\nabla} \phi} \\
\rho\left(\widetilde{\gamma}+\underline{i n \Omega_{0}}\right) \vec{v}_{1} & =-\vec{\nabla} p_{1}+\vec{\jmath}_{1} \times \vec{B}_{0}+\vec{\jmath}_{0} \times \vec{b}_{1}-\underline{\vec{\nabla} \cdot \vec{\Pi}_{1}}-\underline{\rho_{0} \vec{U}} \\
\vec{\jmath}_{1} & =\vec{\nabla} \times \vec{b}_{1}
\end{aligned}
$$

where $\widetilde{\gamma}=\gamma-i \omega$ is the complex growth rate. In Eq. (12),

$$
\vec{U}=\vec{v}_{1} \times\left(\frac{\vec{v}_{0}}{R} \times \widehat{R}\right)+\left(\vec{v}_{1} \cdot \vec{\nabla}\right) \vec{v}_{0}
$$

and

$$
-\vec{\nabla} \cdot \vec{\Pi}_{1}=\text { perturbed viscous force } .
$$

Comparing Eqs. (10) through (15) with the usual linearized MHD equations without flow, we note the presence of the underlined additional terms. These are the Doppler shifts, the modification to Ohm's law in Eq. (11), and the Coriolis force and the perturbed viscous force in Eq. (12). To describe the interaction of the plasma with the resistive wall on the slow time scale, it is crucial that the perturbed viscous force term be included. However, there does not exist a comprehensive theory for 
the proper form or magnitude of this term. We, therefore, have used several different forms to examine the sensitivity of the results to any specific model. These forms are given in the Appendix. One criterion that we used in adopting these forms is that the added terms are dissipative in nature

$$
\int \vec{v} \cdot \vec{\nabla} \cdot \stackrel{\leftrightarrow}{\Pi} d^{3} x>0
$$

The perturbed MHD equations constitute a set of ten coupled linear homogeneous equations for the ten perturbed quantities $\left(\vec{v}_{1}, \vec{b}_{1}, \vec{\jmath}_{1}, p_{1}\right)$. One of the special features in this formulation is that explicit derivatives with respect to the radial variable appear only in first order. The components of $\vec{\jmath}_{1}$ in Eq. (13) are therefore also included explicitly as dependent variables. Not shown explicitly are equations which relate the electric field to the currents in the external wall surrounding the plasma. Equations (10) through (13) form a complex eigenvalue problem for the complex growth rate $\tilde{\gamma}$. Equilibrium flow terms cause the problem to be non-selfadjoint. The MARS code ${ }^{11}$ has been adapted to solve this eigenvalue system. 


\section{Physical Characteristics of the Problem}

Before examining results from the MARS code, we attempt to gain a better understanding of the analytic structure of this system by considering two simple model dispersion relations.

We consider a tokamak plasma surrounded by an external resistive wall located at cylindrical radius $b$ and with a flux diffusion time $\tau_{\mathrm{w}}$. In here $\tau_{\mathrm{w}}$ stands for $\mu_{0} \sigma b \delta$ for a thin wall of thickness $\delta$ and conductivity $\sigma$. If the plasma is static and the external wall perfectly conducting $\left(\tau_{\mathrm{w}}=\infty\right)$, the plasma is assumed to be stable if $b$ is less than a critical distance $b_{c}$. If the plasma is static and the external wall resistive $\left(\tau_{\mathrm{w}}<\infty\right)$, the plasma is unstable to an external mode regardless of the value of $b$.

This stability picture is modified when the plasma rotates with rotation frequency $\Omega_{0}$. If the wall is resistive and $\Omega_{0}$ is large enough, the plasma may be stabilized if $b$ is less than $b_{c}$. This phenomenon of stabilization by rotation occurs differently depending upon whether the plasma mode is a resistive instability (tearing mode) or an ideal external kink. The major difference is that for a tearing mode, the plasma kinetic energy is negligible whereas for an ideal external kink it is not.

First consider the tearing mode case. Starting from the set of equations (10) through (13), specializing first to $\Omega_{0}=0$, and ignoring the plasma inertia, a dispersion relation for tearing mode unstable plasma surrounded by an external perfectly conducting wall may be obtained. A model dispersion relation for the perfectly conducting wall at $b$ and a critical wall location at $b_{\mathrm{T}}$ may be given as $b \tilde{\gamma} \tau_{\eta}+1-b / b_{\mathrm{T}}=0$. Here, $\widetilde{\gamma}$ is the complex growth rate $(\gamma-i \omega)$ and $\tau_{\eta}$ is the tearing mode growth time. The coupling of this tearing mode to the diffusion of flux through an external resistive wall modifies the dispersion relation to $\left(b \tilde{\gamma} \tau_{\eta}+1-b / b_{\mathrm{T}}\right)\left(1+\widetilde{\gamma} b \tau_{\mathrm{w}}^{*}\right)=1$. The quantity $\tau_{\mathrm{w}}^{*}$ is a normalized ${ }^{12}$ resistive wall time. It is $\sim \tau_{\mathrm{w}}(b-1)$ when $b<1+1 / 2 m$, and $\sim \tau_{\mathrm{w}} / 2 m$ when $b>1+1 / 2 m$. Here $m$ is the poloidal mode number. Now there are two roots of the dispersion relation and one of them is always unstable. When $b$ is less than $b_{\mathrm{T}}$, the instability is a result of the coupling to the flux diffusion through the resistive wall and may thus be called a resistive wall mode. With the inclusion of plasma rotation, the dispersion relation is further modified to 


$$
\left[b\left(\widetilde{\gamma}+i n \Omega_{0}\right) \tau_{\eta}+1-\frac{b}{b_{\mathrm{T}}}\right]\left[1+\widetilde{\gamma} \tau_{\mathrm{w}}^{*}\right]=1
$$

A dispersion relation similar to Eq. (17) has been studied before by Bondeson and Persson. ${ }^{13}$ This is a simple quadratic equation in $\widetilde{\gamma}$ and can be easily solved. The characteristics are slightly different depending on whether $\tau_{\mathrm{w}}^{*}$ is much longer or shorter than $\tau_{\eta}$. In present and future tokamaks, we expect $\tau_{\eta}$ to be larger than $\tau_{\mathrm{w}}^{*}$. The typical behavior of the growth rate as a function of $\Omega_{0}$ and the wall position is shown in Fig. 1a. When $\Omega_{0}$ is small (curves marked by 0 ), we have two widely separated branches. One is unstable at any location of the wall $b$ and is called the tearing mode (when $b>b_{\mathrm{T}}$ ) or the resistive wall mode (when $b<b_{\mathrm{T}}$ ). The second mode, in which the perturbed flux is mainly diffusing through the external wall, is stable. As $\Omega_{0}$ increases, these two branches come together. A stability window in $b$ appears near the plasma edge when $\Omega_{0}$ reaches a certain value $\Omega_{0}^{c}$ (curves are marked by $\diamond$ ). The stability window increases in size with $\Omega_{0}>\Omega_{0}^{c}$ (curves are marked by $+)$ and the largest stable $b$ approaches $b_{\mathrm{T}}$ as $\Omega_{0}$ tends to $\infty$. Note that we showed only the growth rate (real part of $\widetilde{\gamma}$ ) in Fig. 1a. The full solution also shows that the unstable branch has its frequency $\omega$ (imaginary part of $\widetilde{\gamma}=\gamma-i \omega$ ) always close to $\Omega_{0}$ (locked to the plasma), whereas the stable branch has $\omega$ always very small (locked to the wall).

If the tearing mode growth time is much shorter than the resistive wall time $\left(\tau_{\eta} \ll \tau_{w}^{*}\right)$, the growth rate diagram is as shown in Fig. 1b. Here, below a critical $\Omega_{0}$, the two stability branches are widely separated as shown in Fig. 1a. But as $\Omega_{0}$ is increased, the stability window first appears at a location intermediate between the plasma edge $(b=1)$ and $b_{\mathrm{T}}$. A further increase in $\Omega_{0}$ widens this stability window. As $\Omega_{0}$ increases, the frequency of the unstable mode does not increase with $\Omega_{0}$. Rather, it remains very close to zero (locked to the wall). The frequency of the stable branch, however, is always close to the $\Omega_{0}$ (locked to the plasma).

If the plasma behavior is close to ideal, then the kinetic energy term is of importance. Starting from Eqs. (10) through (14) in Section II, we may derive the following dispersion relation for a plasma with a uniform rotation at angular frequency $\Omega_{0}$

$$
\left(\widetilde{\gamma}+i n \Omega_{0}\right)^{2} K+\left(\widetilde{\gamma}+i n \Omega_{0}\right) D+\delta W_{\mathrm{p}}+\frac{\delta W_{\mathrm{v}}^{b} \widetilde{\gamma} \tau_{\mathrm{w}}^{*}+\delta W_{\mathrm{v}}^{\infty}}{\widetilde{\gamma} \tau_{\mathrm{w}}^{*}+1}=0
$$




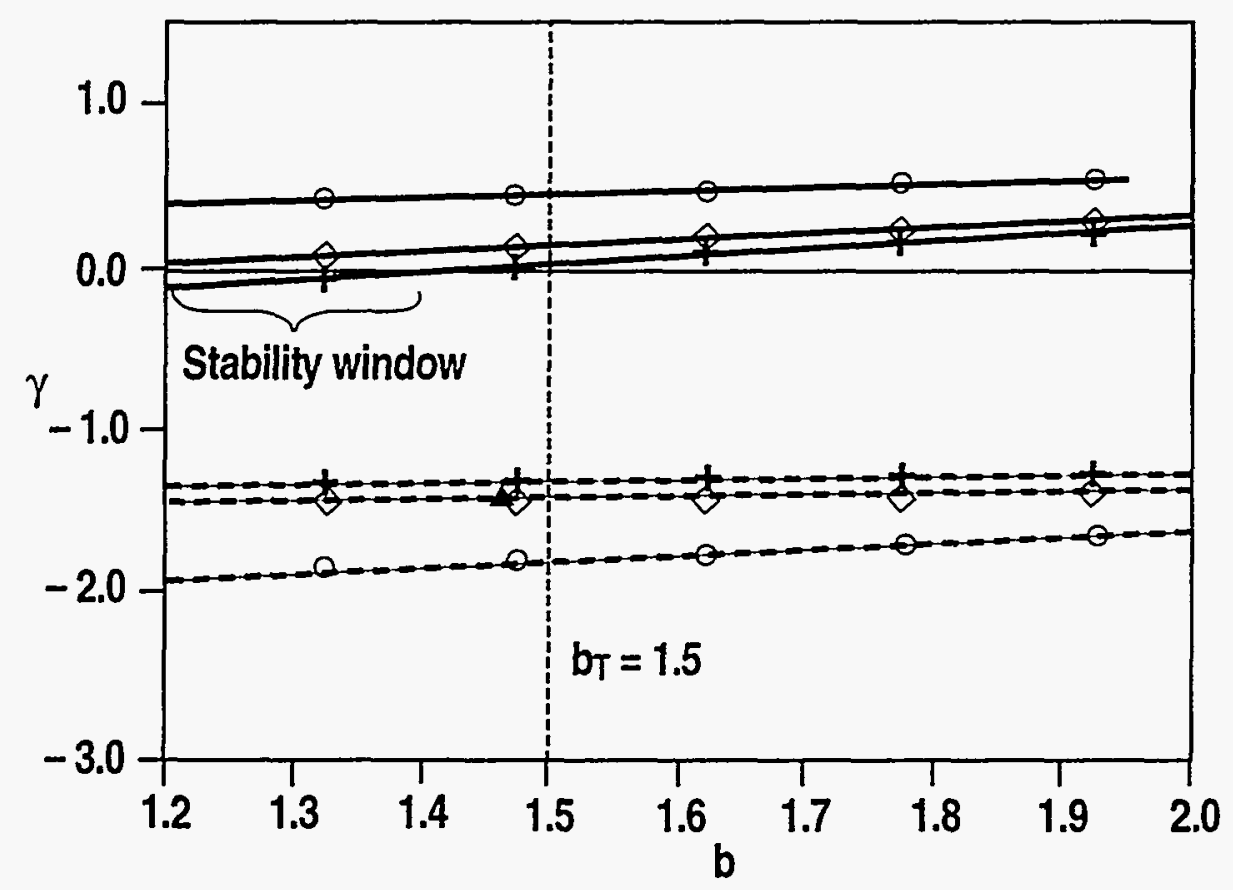

FIG. 1a. Growth rate $\gamma$ as a function of external wall location $b$ for coupled tearing mode and diffusion through the resistive wall, Eq. (17). The critical wall distance is at $b_{\mathrm{T}}=1.5$. The two branches are coupled by plasma rotation $\Omega_{0}$. As $\Omega_{0}$ increases, a stability window first appears near the plasma edge. This is the case for $\tau_{\mathrm{w}} \ll \tau_{\eta}$.

A similar dispersion relation has also been obtained by Betti and Freidberg ${ }^{14}$ and Fitzpatrick. ${ }^{15}$

In (18), $K$ is the kinetic energy integral, $D$ is the dissipation energy integral, $\delta W_{\mathrm{p}}$ is the plasma potential energy, $\delta W_{\mathrm{v}}^{b}$ is the vacuum energy integral with a perfectly conducting wall at location $b$ and $\delta W_{\mathrm{v}}^{\infty}$ is the vacuum energy with the perfectly conducting wall at infinity. It is assumed that for a perfectly conducting wall $\left(\tau_{\mathrm{w}}^{*}=\infty\right)$, the plasma is stable when $b$ is less than $b_{\mathrm{c}}$. From Eq. (18), this indicates that $\delta W_{\mathrm{p}}$ is equal to $-\delta W_{\mathrm{v}}^{b_{\mathrm{c}}}$ when $b$ equals $b_{\mathrm{c}}$. As $b$ goes to $\infty$, the system is unstable with $\delta W_{\mathrm{p}}+\delta W_{\mathrm{v}}^{\infty}<0$.

We see that in contrast to the quadratic equation in $\widetilde{\gamma}$ for the tearing mode given by Eq. (17), the dispersion relation for the coupled external kink and resistive wall gives rise to a cubic equation, Eq. (18). Without rotation, and with a perfectly conducting external wall, there are two modes present in the plasma. One is the 


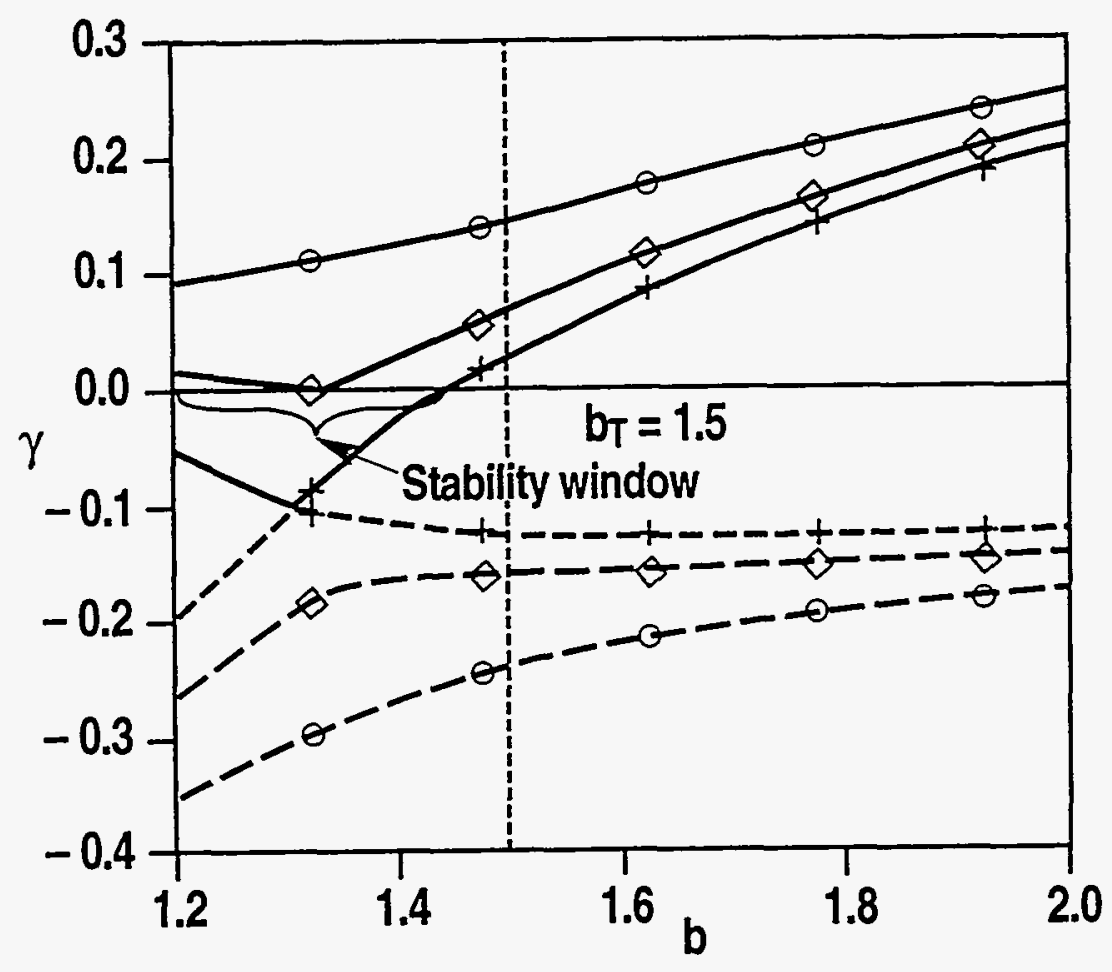

FIG. 1b. Same as Fig. 1a for the case of $\tau_{\eta} \gg \tau_{\mathrm{w}}$. The stability window first appears at the location between the plasma edge $b=1$ and $b_{\mathrm{T}}$.

unstable external kink when $b$ is larger than $b_{c}$ and the other is a damped (stable) plasma mode that may be modified by plasma effects not included in this model. Resistive diffusion of the flux introduces a third branch into the stability diagram and destabilizes the external kink when $b$ is less than $b_{c}$. It appears as the resistive wall mode. This is indicated in Fig. 2. As $\Omega_{0}$ increases, the resistive wall mode is stabilized by coupling to the wall mode. This mode coupling is indicated in Fig. 2 by the curves $\Omega_{0}=\Omega_{0}^{c}$. At $\Omega_{0}^{c}$, the two branches coalesce and exchange character (note this is not the rotation frequency at which the stability window first appears). A further increase in $\Omega_{0}$ leads to further opening up of the stability window. [Note that without dissipation, the solution of (18) indicates the damped branch does not couple with the resistive wall mode. No stabilization is achieved by increasing $\Omega_{0}$ when there is no dissipation.] 


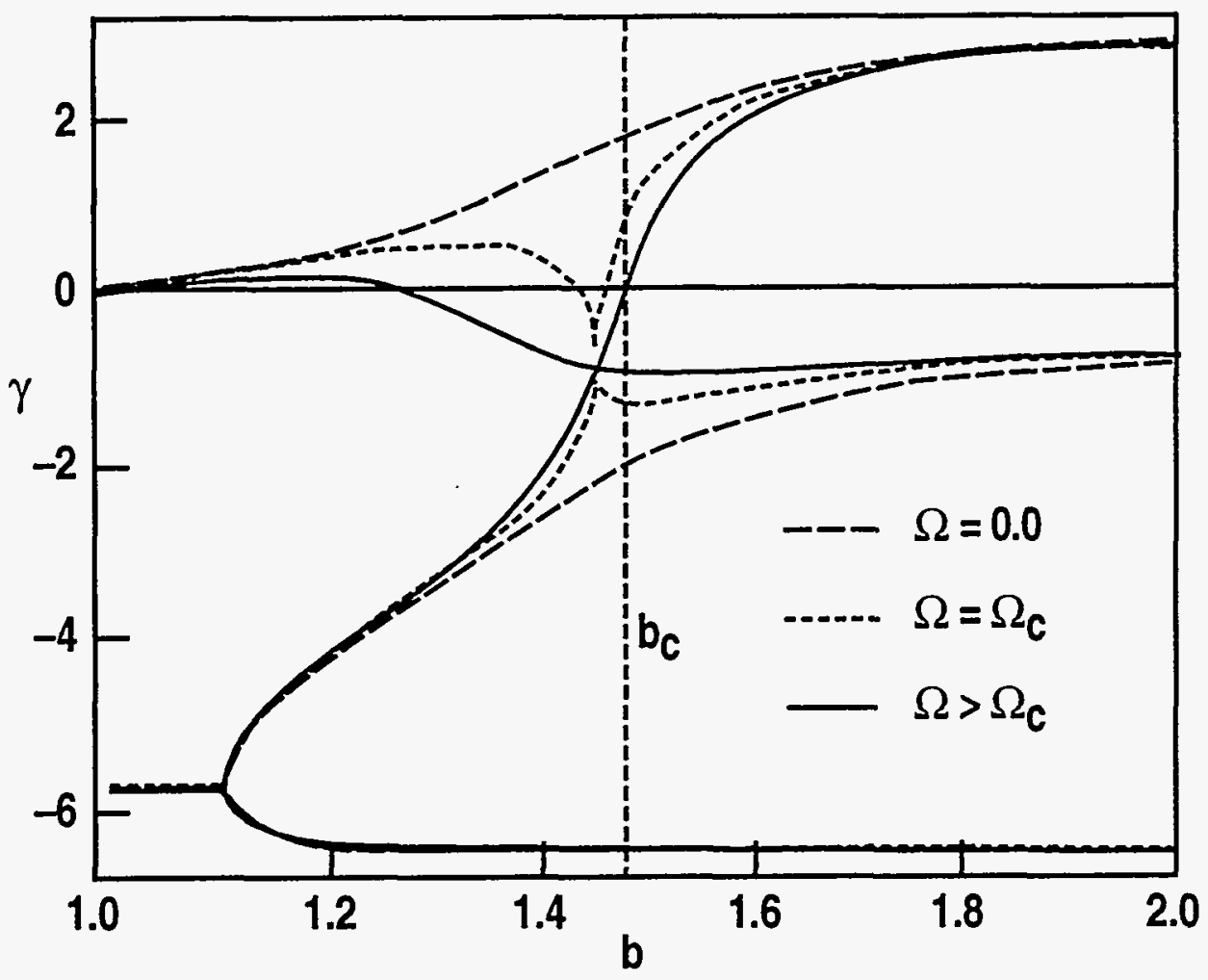

FIG. 2. Growth rate $\gamma$ as a function of the external wall location $b$ for coupled external kink and diffusion through the resistive wall, Eq. (18). The critical wall distance is at $b_{c}=1.6$. Three branches of the solution are shown. At $\Omega_{0}$ (no plasma rotation, long dashed curves), one of the branches (external kink-resistive wall mode) is unstable at all $b$. The other two branches are stable. As $\Omega_{0}$ increases, induced coupling between the stable and unstable branch causes the stability window to appear. $\Omega_{0}^{c}$ is the critical frequency for mode coupling. It is also close to the frequency at which the stability window first appears. The three branches of solutions at $\Omega_{0}^{c}$ are shown as dotted curves. For $\Omega>\Omega_{0}^{c}$, the stability windows become substantial in size. These three branches are shown as solid curves.

We thus see that the combined presence of plasma dissipation and plasma rotation profoundly changes the topology of the three roots of the dispersion relation. While rotation alone separates the resistive wall mode from the "ideal" plasma mode, dissipation reduces the growth rate of the resistive wall mode and also imparts a finite real frequency to it. For a large enough rotation, and when $D$ exceeds a minimum 
value, the plasma stability for a fixed external wall then approaches that determined for a static plasma surrounded by a perfectly conducting wall. 


\section{Numerical Results}

In this section we describe the numerical results obtained by applying the adapted MARS code to high beta equilibria. We begin with one of the cases that was studied previously by Bondeson and Ward. ${ }^{4}$ This equilibrium has aspect ratio $R / a=3$, elongation $\kappa=1.6$, triangularity $\delta=0.3, q_{0}=1.2$, edge safety factor $q_{\mathrm{e}}=2.55$, plasma $\beta=6.7 \%$, normalized current $I_{\mathrm{N}}=I / a B=1.6$, and normalized $\beta_{\mathrm{N}}=\beta a B / I=4.25$. Note that there is only one resonant surface in the plasma. In contrast to Bondeson and Ward, ${ }^{4}$ here the plasma is rotating instead of the external wall; the effects due to the Coriolis force and flow shear can thus be studied. We normalize the rotation frequency to the Alfvén frequency $\Omega_{\mathrm{A}} \equiv v_{\mathrm{A}} / R$, where $v_{\mathrm{A}}$ is the Alfvén velocity at the plasma center and $R$ is the major radius. In this first example, the plasma has a uniform rotation frequency of $0.06 \Omega_{\mathrm{A}}$. By varying the position of the external resistive wall (with $\Omega_{\mathrm{A}} \tau_{\mathrm{w}}=5000$ ), three distinct branches of the mode growth rates are found as shown in Fig. 3. The curve labeled $\gamma_{\mathrm{I}}$ is the ideal branch; it has a frequency $\omega$ close to $\Omega_{0}$. The resistive wall mode branch labeled $\gamma_{\mathrm{w}}$ has frequency close to zero. The third branch, labeled $\gamma_{3}$, is always heavily damped. This branch also has a very small frequency. The ideal branch and the resistive wall branch have very similar eigenfunctions within the plasma whereas the third branch has a different eigenfunction. Comparing these results with the expected analytic structure from Eq. (18), we recognize that another damped branch with its frequency $\omega$ close to $\Omega_{0}$ is not found in the present numerical scheme. Also, the coupling of different components of the mode amplitudes can give rise to new modes with different polarizations in addition to those predicted by the simple model of Eq. (18). We have also found that the Coriolis force term in Eq. (12) has negligible effect. This justifies a posteriori the neglect of higher order plasma flow terms in the equilibrium equation. We see that in this case a stability window exists for $b_{1}=1.3<b<b_{\mathrm{c}}=1.66$.

The stability window shown in Fig. 3 may be altered by varying the plasma rotation frequency or the amount of dissipation. It is found that the plasma has to be rotating faster than a threshold value $\Omega_{0}^{t}$ for the stability window to exist. And in general, $\Omega_{\mathbf{0}}^{\mathrm{t}}$ decreases and the width of the stability window increases with increasing dissipation. ${ }^{16}$ Shown in Fig. 4 are computations illustrating these dependences for the same plasma configuration shown in Fig. 3. Case A has $\Omega_{0}=0.04 \Omega_{\mathrm{A}}, \kappa_{\|}=1$ [the 


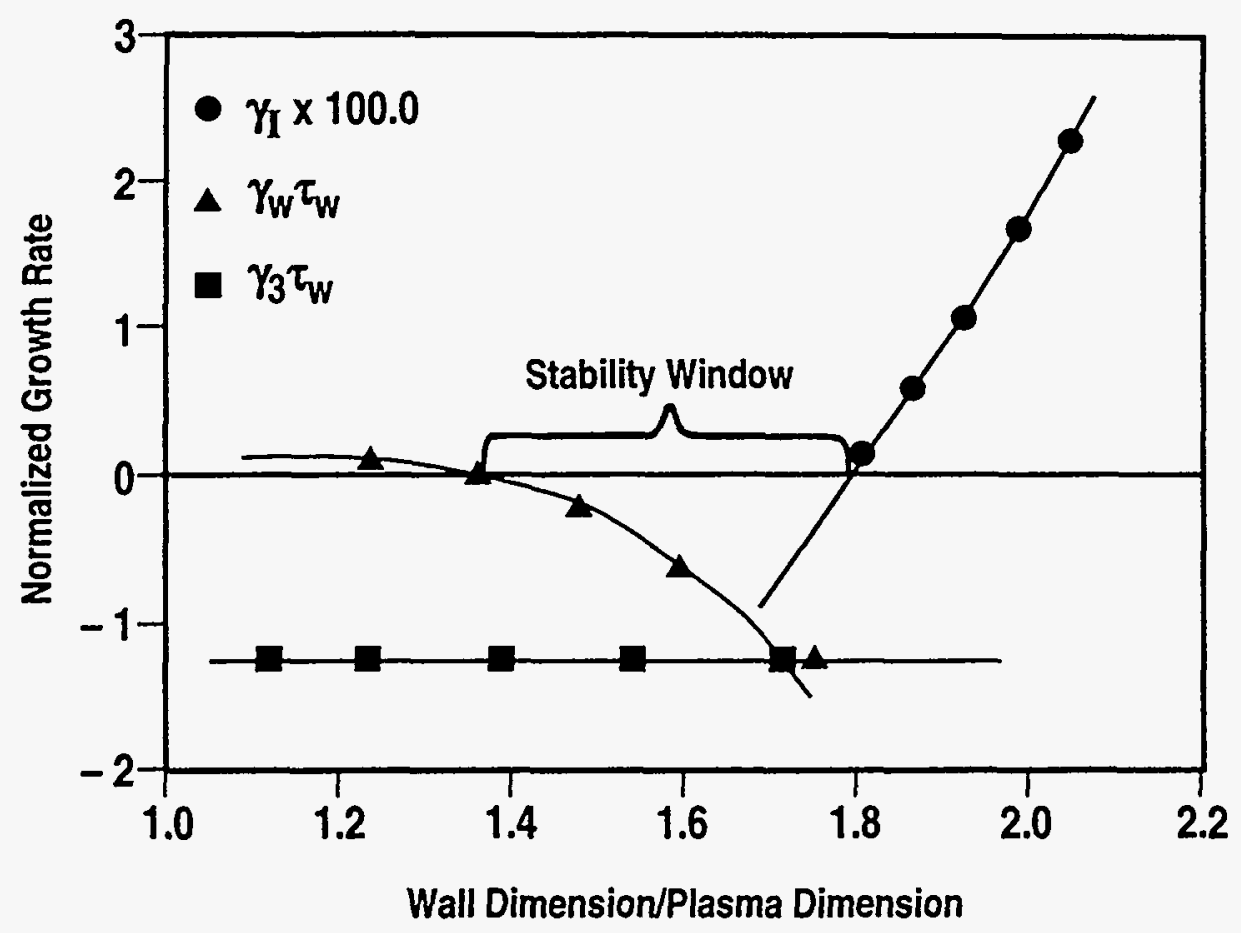

FIG. 3. The growth rate diagram computed for a low edge $q$ configuration. Shown are the three branches of the solution for a numerical high $\beta$ equilibrium with $R / a=3, \kappa=1.6, q_{0}=1.2$, $q_{\mathrm{e}}=2.55, \beta=6.7 \%, I / a B=1.6, \beta_{\mathrm{N}}=4.25$. The plasma is assumed to have a uniform rotation at $\Omega_{0}=0.06 \Omega_{\mathrm{A}}$. The wall is assumed to be $\tau_{\mathrm{w}}=5000 \tau_{\mathrm{A}}$.

definition of $\kappa_{\|}$is given in Eq. (A2)], case $\mathrm{B}$ has $\Omega_{0}=0.06 \Omega_{\mathrm{A}}, \kappa_{\|}=0.1$. The values of $\eta$ and $\mu_{\perp}$ are 0 for both A and B. Case C has $\Omega_{0}=0.06 \Omega_{\mathrm{A}}, \kappa_{\|}=1, \eta=10^{-6}$, $\mu_{\perp}=7 \times 10^{-5}$. These transport coefficients are measured in units of global transport rate in time units of $\tau_{\mathrm{A}}$. We have also varied the resistivity of the plasma and find that it does not change the general nature of the stability diagram; thus, this resistive wall mode results from the coupling to the external kink rather than to a tearing mode. ${ }^{17}$

Shown in Fig. 5 is the effect of flow shear for this configuration. We compare the growth rates of a uniformly rotating plasma with those of a plasma with sheared rotation. For uniform rotation, the rotation frequency at the $q=2$ surface is the same as that at the plasma center. For the sheared rotation, we used the profile $\Omega_{0}=\Omega_{0}^{(0)}\left[1-s_{\mathrm{v}} \rho^{2}(1-2 / 3 \rho)\right]$ where $\rho$ is a generalized plasma radius defined as $(\widetilde{\psi})^{1 / 2}, \widetilde{\psi}$ is the normalized poloidal flux, and $\rho=\widetilde{\psi}=1$ at the plasma boundary. 


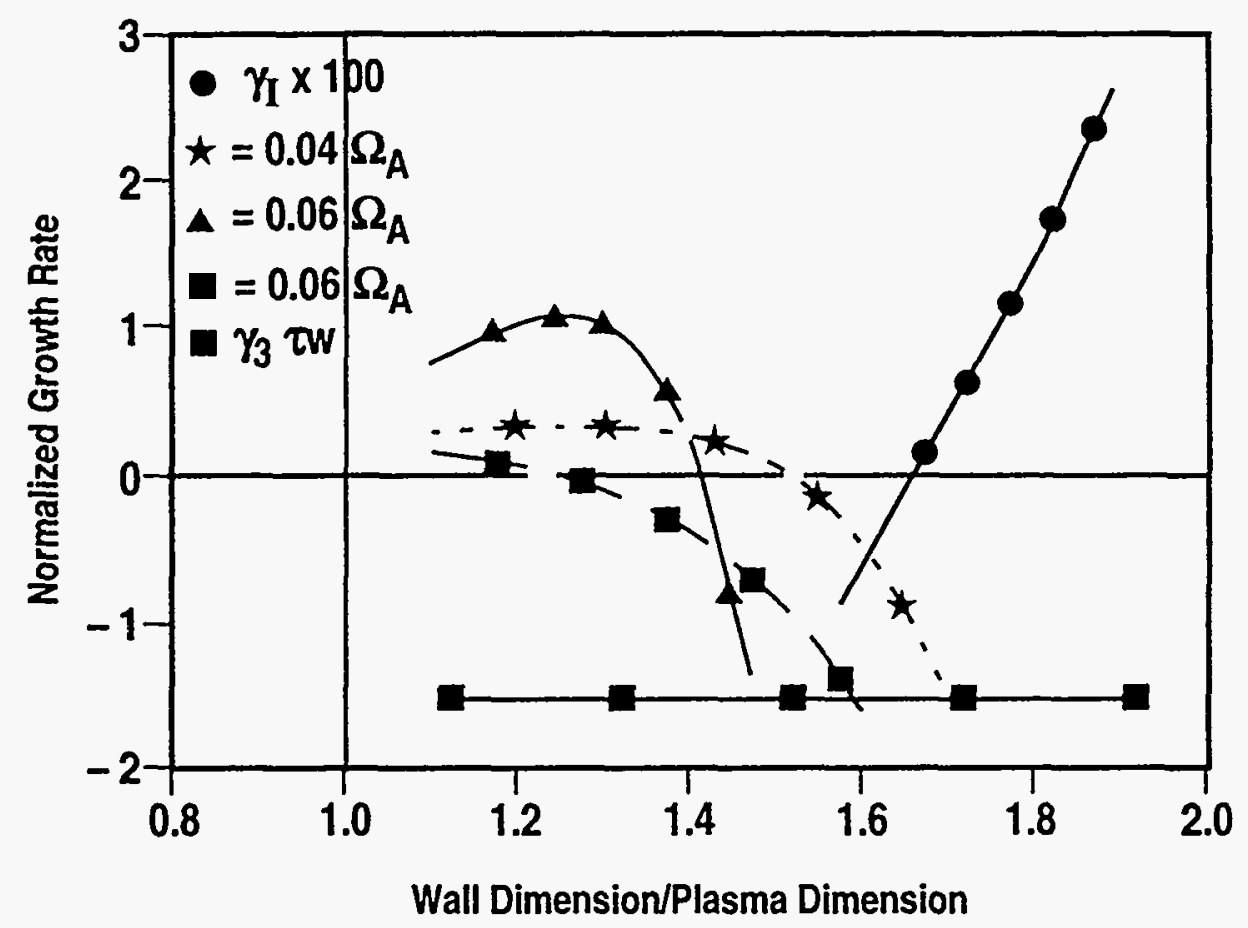

FIG. 4. The stability diagram for the same equilibrium shown in Fig. 3 with different rotation frequency and plasma dissipation. This shows that the stability window is enlarged by both increase in plasma rotation frequency and increase in dissipation. In case $A$, $\Omega_{0}=0.04 \Omega_{\mathrm{A}}, \kappa_{\|}=1$; case $\mathrm{B}, \Omega_{0}=0.06 \Omega_{\mathrm{A}}, \kappa_{\|}=0.1$; case $\mathrm{C}$, $\Omega_{0}=0.06 \Omega_{\mathrm{A}}, \kappa_{\|}=1, \eta=10^{-6}, \mu_{\perp}=7 \times 10^{-5}$.

The rotation frequency is held fixed at $0.06 \Omega_{\mathrm{A}}$ at the plasma center. The rotation velocity at the $q=2$ surface is decreased by increasing $s_{\mathrm{v}}$. The growth rates are plotted as a function of the rotation speed of the $q=2$ surface with respect to the external wall. It is seen that, for this configuration, the rotation frequency at the $q=2$ surface is more important than $s_{\mathrm{v}}$ in determining the stability of the resistive wall mode.

Shown in Fig. 6 is the stability diagram for an equilibrium simulating a DIII-D discharge with $R / a=2.49, \kappa=2.1, \delta=0.8, q_{0}=1.405, q_{\mathrm{e}}=5.8, \beta=6 \%$, $I_{\mathrm{N}}=1.6, \beta_{\mathrm{N}}=3.8, \Omega_{0} / \Omega_{\mathrm{A}}=0.01, \Omega_{\mathrm{A}} \tau_{\mathrm{w}}=5000$, and $\kappa_{\|}=1$. There is no rotation shear. Once again, the stability diagram is that of an unstable external kink mode when no wall is present. When we vary the plasma rotation speed, keeping the experimentally obtained density and rotation profiles, we find the stability threshold in rotation is similar to that observed in the experiment. In this comparison, we 


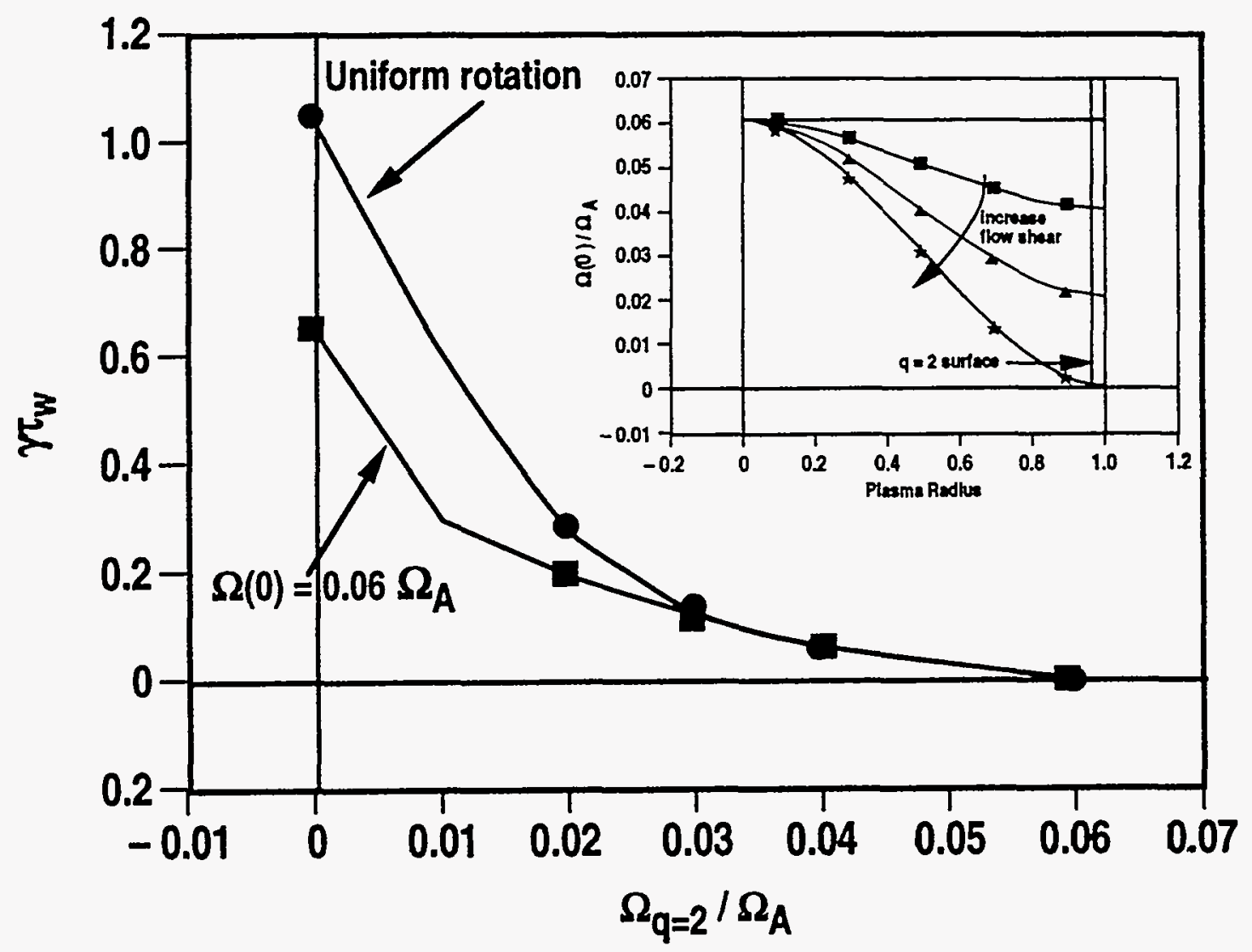

FIG. 5. The growth rate plotted as a function of the rotation frequency of the $q=2$ surface for rotation profiles with and without rotation shear. The growth rate for uniformly rotating plasma is slightly higher than that of a plasma with rotation shear, showing that rotation at the singular surface is more important for stabilization than rotation shear.

have taken $\kappa_{\|}=0.5$. This variation with rotation is shown in Fig. 7. Although in the experimental case, near the plasma edge, Eq. (6) is not fully satisfied, we do not expect the present conclusion to be modified by the inclusion of inertial effects in the equilibrium. This is also being studied presently. 


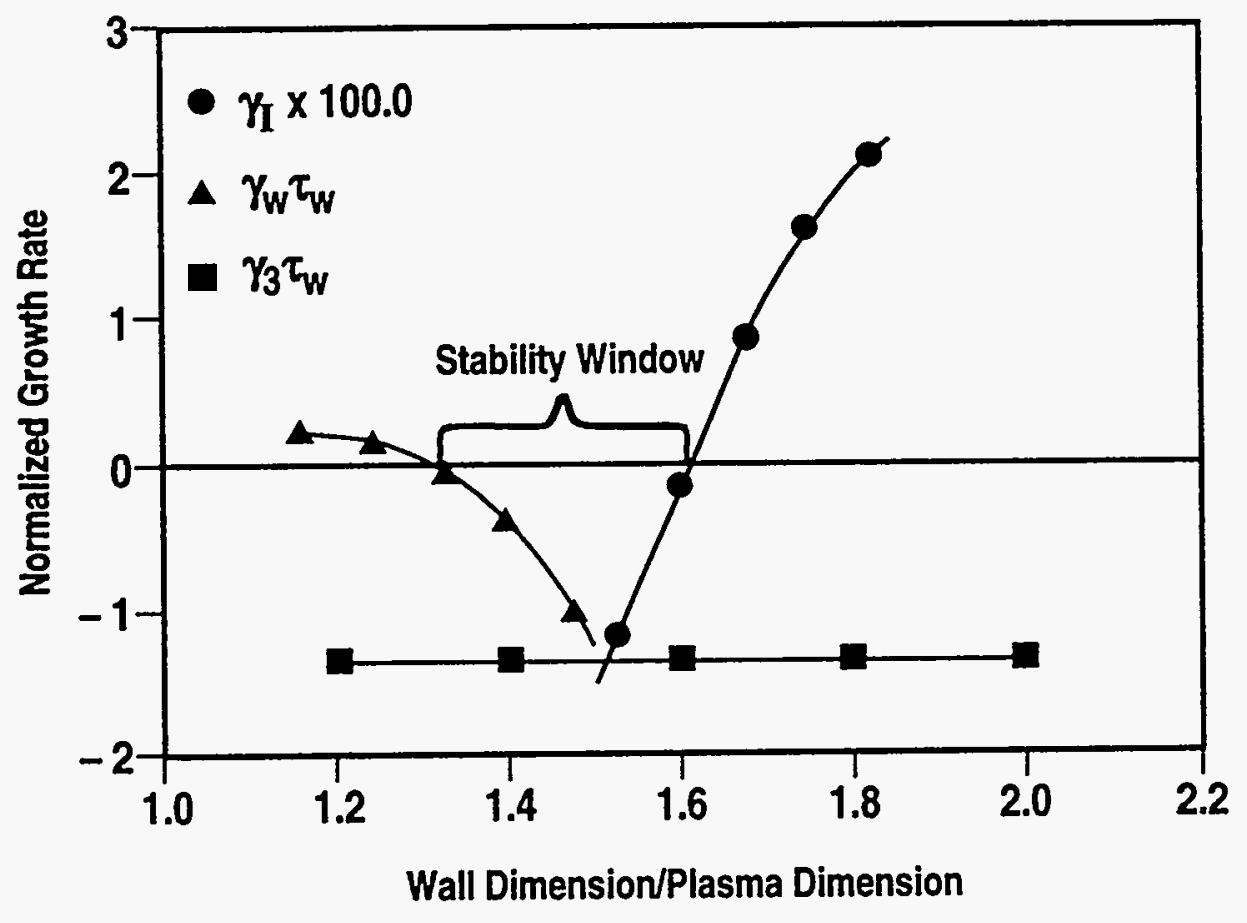

FIG. 6. Stability diagram for a high edge $q$ configuration, simulating a discharge in DIII-D. This equilibrium has $R / a=2.49$, $\kappa=2.1, \delta=0.8, q_{0}=1.405, q_{\mathrm{e}}=5.8, \beta=6 \%, I / a B=1.6$, $\beta_{\mathrm{N}}=3.8, \kappa_{\|}=1$. The plasma is assumed to be rotating at $\Omega_{0}=0.01 \Omega_{\mathrm{A}}$ and $\tau_{\mathrm{w}}=5000 \tau_{\mathrm{A}}$. 


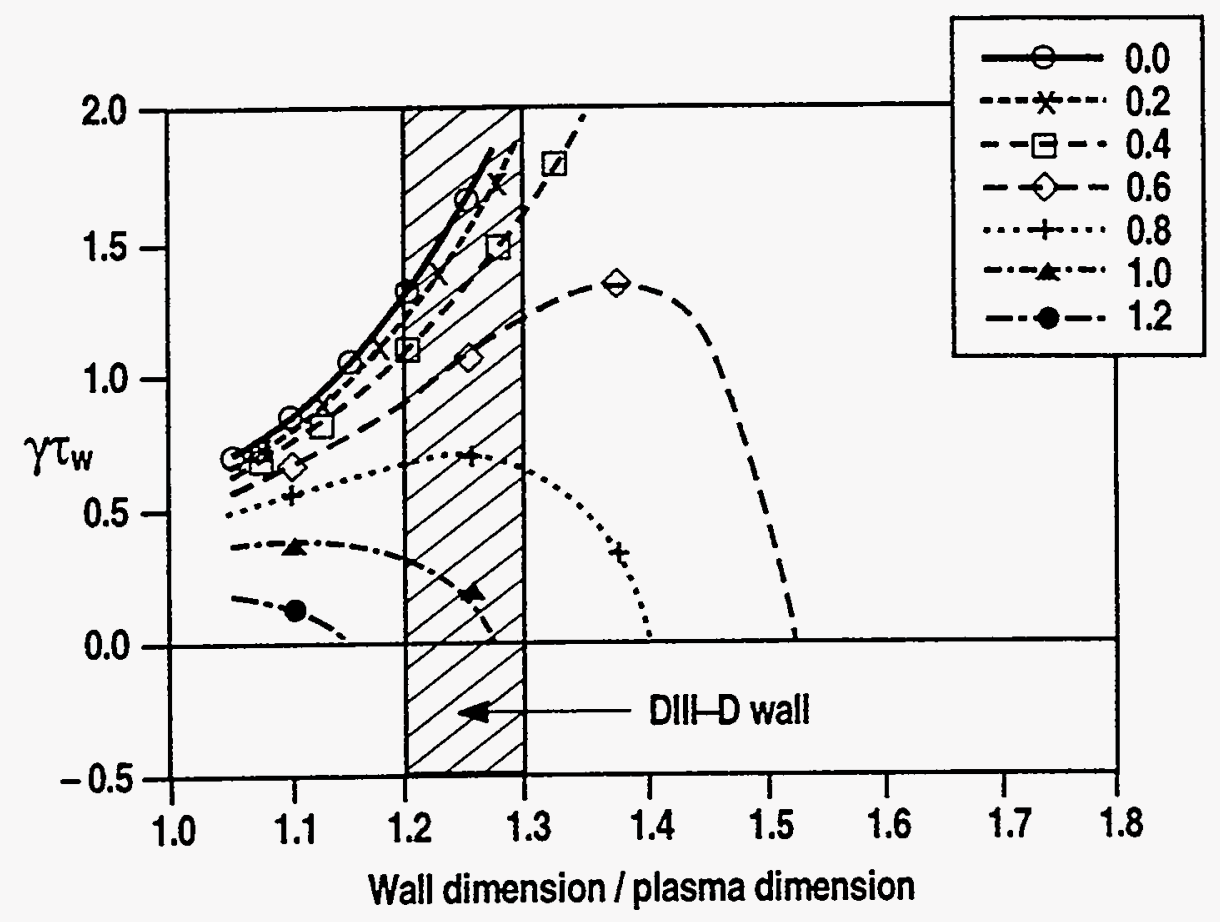

FIG. 7. Stability diagram for the same equilibrium as shown in Fig. 6 with fitted profiles to experimental rotation and density profiles. Computed are growth rates for different levels of rotation frequency and with $\kappa_{\|}=0.5$. It is shown that the experimentally observed threshold is well within the computed range of values. 


\section{Conclusion and Summary}

In this paper, we have formulated the MHD stability problem for a tokamak plasma with a subsonic toroidal flow, surrounded by an external resistive wall. For weak toroidal flow, the plasma satisfies the Grad-Shafranov equation without flow. Linear perturbations satisfy equations that constitute a non-self-adjoint eigenvalue problem for which the complex growth rate is the eigenvalue. The equations contain the usual MHD equations with additional terms coming from the Doppler shift, modification to Ohm's law, Coriolis force terms, and viscous dissipation. The complex eigenvalue problem is solved using an extension of the MARS code.

When a static unstable plasma is surrounded by an external resistive wall, diffusion of the perturbed flux through the external wall will make the plasma unstable at any location of the resistive wall. Plasma rotation can couple the unstable mode with the stable damped mode and stabilize the plasma. Two simple dispersion relations are used to illustrate the characteristics of the solutions. Depending on the nature of the plasma response, the coupled dispersion relation is either quadratic for the tearing mode or cubic for the ideal external kink mode. The general features of these solutions are give in Section III.

The characteristics of the coupled system of an unstable external kink with a resistive wall (the resistive wall mode) are verified by computational examples of realistic equilibria for both a low $q_{\mathrm{e}}$ and a high $q_{\mathrm{e}}$ plasma. In general, it is found that sufficient toroidal flow opens up a stability window for the external wall location. The window size is enhanced by an increased flow velocity or an increased amount of plasma dissipation. We find the effect of the Coriolis force and other inertial forces on these modes to be minimal. For the low $q_{\mathrm{e}}$ example, we find that stability depends more on the velocity of the resonant $q$ surface relative to the external wall rather than on the flow shear. For the high $q_{\mathrm{e}}$ example which simulates a DIII-D discharge, we find that the experimentally observed rotation speed is sufficient for the stabilization of the resistive wall mode. Insufficient knowledge of the plasma viscosity tensor from first principles means that the dissipation can be adjusted somewhat to favorably compare with experiment. This does not impact the favorable qualitative comparison with experiment but it makes the quantitative comparison less sharp. 


\section{Acknowledgements}

The authors would like to acknowledge discussions with Drs. R. Betti, L. Chen, R. Fitzpatrick, Y.-B. Kim, and K. Shaing. They would also like to acknowledge Drs. L.L. Lao, E.J. Strait, T.S. Taylor, and A.D. Turnbull for clarification of features observed in experiments.

This is a report of work sponsored by the U.S. Department of Energy under Contract No. DE-AC03-89ER51114. 


\section{References}

${ }^{1}$ D. Pfirsch and H. Tasso, Nucl. Fusion 11, 259 (1971).

2J.P. Freidberg, Ideal Magnetohydrodynamics (Plenum Press, New York, 1987), p. 309.

${ }^{3}$ T.H. Jensen and M.S. Chu, J. Plasma Phys. 30, 57 (1983).

${ }^{4}$ A. Bondeson and D.J. Ward, Phys. Rev. Lett. 72, 2709 (1994).

5J.L. Luxon and L.G. Davis, Fusion Technol. 8, 441 (1985).

${ }^{6}$ T.S. Taylor, E.J. Strait, L.L. Lao, M.E. Mauel, A.D. Turnbull, K.H. Burrell, M.S. Chu, J.R. Ferron, R.J. Groebner, R.J. La Haye, B.W. Rice, R.T. Snider, S.J. Thompson, D. Wròblewski, and D.J. Lightly, "Wall Stabilization of High Beta Plasmas in DIII-D," to be published in Physics of Plasmas.

${ }^{7}$ A.D. Turnbull, T.S. Taylor, E.J. Strait, S.J. Thompson, M.S. Chu, J.R. Ferron, R.J. La Haye, L.L. Lao, R.T. Snider, B.W. Rice, D. Wròblewski, O. Sauter, M.E. Mauel, A. Popov, N. Popov, D.J. Lightly, and J.D. Williams, "Wall Stabilization of Rotating High Beta Discharges in DIII-D," in Plasma Physics and Controlled Nuclear Physics Research 1994 (Proc. 15th Int. Conf. Seville) (International Atomic Energy Agency, Vienna, to be published) paper IAEA-CN-60/A-5-11-4.

${ }^{8}$ R. Fitzpatrick, R.J. Hastie, T.J. Martin, and C.M. Roach, Nucl. Fusion 33, 1533 (1993).

${ }^{9}$ R.L. Dewar and M. Persson, Phys. Fluids B 5, 4273 (1993).

${ }^{10}$ E.A. Frieman and M. Rotenberg, Rev. Mod. Phys. 32, 989 (1960).

${ }^{11}$ A. Bondeson, G. Vlad, and H. Lutjens, Phys. Fluids B 4, 1889 (1992).

${ }^{12}$ S.W. Haney and J.P. Freidberg, Phys. Fluids B 1, 1637 (1989).

${ }^{13}$ A. Bondeson and M. Persson, Nucl. Fusion 28, 1887 (1988).

${ }^{14}$ R. Betti and J.P. Friedberg, "Stability Analysis of Resistive Wall Kink Modes in Rotating Plasmas," submitted to Physical Review Letters.

${ }^{15}$ R. Fitzpatrick, private communications (1994).

${ }^{16}$ N. Pomphrey, S.C. Jardin, J. Bialek, M.S. Chance, D.A. D'Ippolito, J.M. Finn, R. Fitzpatrick, J.L. Johnson, C.E. Kessel, J. Manickam, D.A. Monticello, J.R. Myra, M. Ono, W. Park, A. Reiman, G. Rewolt, W.M. Tang, E.J. Valeo, and L.E. Zakharov, in Plasma Physics and Controlled Nuclear Physics Research 1994 (Proc. 15th Int. Conf. Seville) (International Atomic Energy Agency, Vienna, to be published) paper IAEA-CN-60/D-1-14. 
${ }^{17}$ J.M. Finn, "Resistive Wall Stabilization of External Kinks and Tearing Modes," submitted to Physics of Plasmas (1994).

${ }^{18}$ M. Yagi, J.P. Wang, Y.-B. Kim, and M. Azumi, Phys. Fluids B 5, 1179 (1993). 


\section{Appendix: Viscous Damping Models}

The correct choice for a viscous damping model is unresolved. We therefore list a few of the possibilities here and expect to be able to refine them later on. The viscous force has been taken to be

$$
-\vec{\nabla} \cdot \vec{\Pi}=\overrightarrow{\mathcal{F}}_{\mathrm{S}, \mathrm{D}}+\overrightarrow{\mathcal{F}}_{\text {neo,D }}+\overrightarrow{\mathcal{F}}_{\perp \mathrm{D}}
$$

In (A1), the parallel sound wave damping mode ${ }^{1}$ is

$$
\overrightarrow{\mathcal{F}}_{\mathrm{SD}}=-\kappa_{\|} \sqrt{\pi}\left|k_{\|} v_{\mathrm{thi}}\right| \rho \vec{v}_{1} \cdot \widehat{b} \widehat{b}
$$

where $k_{\|}$is the parallel wavenumber $(n-m / q)(1 / R)$, and $\kappa_{\|}$is the strength parameter. This is used to model the Landau damping effect. The neoclassical damping model used is adapted from Ref. 18,

$$
\overrightarrow{\mathcal{F}}_{\text {neo }}=\kappa_{\text {neo }}\left[2 \rho \nu_{\text {ii }} \mu_{1} q^{2} \frac{R^{2}}{\rho^{2}} \vec{v}_{1} \cdot \widehat{R}\right] \widehat{R}
$$

with $\nu_{\mathrm{ii}}$ the ion collision frequency and $\mu_{1}$ defined in Ref. 16. $\kappa_{\text {neo }}$ is the strength parameter for this model. The force from anomalous perpendicular viscosity is taken to be

$$
\overrightarrow{\mathcal{F}}_{\mu_{\perp} \mathrm{D}}=-\left[\vec{\nabla} \times \rho \mu_{\perp} \vec{\nabla} \times \vec{v}_{1_{\perp}}\right]_{\perp}
$$

Three adjustable parameters $\left(\kappa_{\|}, \kappa_{\text {neo }}, \kappa_{\perp}\right)$ are used within MARS to examine the sensitivity of the various damping models on plasma stability limits. 